\title{
Analysis of Security Issues of Edge Computing Based Internet of Things Applications
}

\author{
Inderveer Chana, Shamim Muhammad, Supriya Thilakanathan \\ Dept Computer Science, Thapar Institute of Enggineering and Technology
}

\begin{abstract}
Edge computing is a technology that allows resources to be processed or executed close to the edge of the internet. The interconnected network of devices in the Internet of Things has led to an increased amount of data, increasing internet traffic usage every year. Also, edge computing is driving applications and computing power away from the integrated points to areas close to users, leading to improved performance of the application. Despite the explosive growth of the edge computing paradigm, there are common security vulnerabilities associated with the Internet of Things applications. This paper will evaluate and analyze some of the most common security issues that pose a serious threat to the edge computing paradigm.
\end{abstract}

Keywords- Edge Computing, Security, Privacy, Internet of Things, IoT

\section{INTRODUCTION}

Internet of Things is the next wave of computing where distributed devices automate common tasks and make management possible through user friendly mobile interface [1]. Many businesses worldwide are embracing computing technology as it is the most efficient and cost-effective solution to meet their needs. Distribution, and decentralization of computing power from a single mainframe to a network of computing nodes e.g., a massive network of servers to improve data processing and storage, are being embraced by many organizations worldwide [2]. Researchers have realized the possibilities of the increasing number of devices being deployed in the internet of things (IoT).

Therefore, edge computing is the act of moving power, decentralizing the network, and other functions away from a single hub into the device around the edge. Recently, edge computing is a major part of the evolution of the Internet of Things. It ensures that data processing and storage are carried out on network edges closer to the Internet of Things users, rather than on centralized points and cloud servers. Therefore, when data, applications, and computing power are operated away from integrated points to locations closer to the user, distribution of storage and power leads to better performance.

Although edge computing improves the efficiency of systems built using Internet of Things devices, there is a large strain on cloud computing servers and communication networks. The reason is that original IoT devices were developed to have a minimum amount of computing power to collect and transmit data and receive and implement instructions. Therefore, a large amount of strain is experienced when processing and transmitting the massive amount of data collected by millions of IoT applications. IoT applications also experience delays as they wait for data to be sent away, servers to process it, and instructions to arrive. This research paper will evaluate some of the most common security issues of edge computing based on IoT applications.

\section{IOT AND EDGE COMPUTING ARCHITECTURE}

There are two categories of computing technology devices, including edge computing devices and edge computing-based IoT devices and technologies. The two categories vary in capabilities and services, but core features, e.g., processing, artificial intelligence model, complex event processing, and data management, differentiate these systems. The edge computing architecture has three levels. The first level holds IoT devices that can communicate with each other remotely using remote pooling techniques before being transferred to edge servers. The second level is referred to as the edge server platform that acts as the access point of the network resources, e.g., computation and storage. These level reduces latency and improves load processing. The third level is the infrastructurebased cloud, where data computation and processing occur. The data generated by sensors and IoT devices are also stored in this level, and any other device of another level can virtually access the data.

It is necessary to understand that the Internet of things has resource-constrained devices, e.g., smartphones, wearable devices, and sensors. Therefore, edge computing provides wide-spread geographical distribution, low latency, mobility support, and location to the IoT applications. Edge computing creates an environment where enterprises can operate using local computing, or a public or private cloud in a specific region that requires local security boundary.

\section{SECURITY ISSUES OF EDGE COMPUTING-BASED INTERNET OF THINGS APPLICATIONS}

\section{A. Privacy}

This is the main challenge in these applications, despite the user's data undergoing data encryption and security routine before being transmitted. Mostly, IoT device users tend to use weak passwords for all the accounts connected to the IoT applications. This poses a huge risk since attackers can crack their passwords and gain access to their accounts. It also common to find IoT applications built with an interface that comes with a default password that users are encouraged to change as part of the operating instructions. Definitely, only a few users who are going to set a unique password. This shows that edge computing-based IoT applications are likely to be protected with weak passwords creating a security threat to the data stored in them. Therefore, 
it would be possible if the developers expand their password policies in the applications.

\section{B. Maintenance and support}

Detection failure, intrusion detection, and device malfunction are the common predictive maintenance that is needed in edge computing. There, it is possible to find areas where IoT devices fail to have a predictive program simply for detection. This hinders edge computing from connecting multiple data sources to the machine data source, leading to an inaccurate data overview. Another common issue is that the Internet of Things devices is mostly designed to stick around for a while without upgrading [5]. Due to the increasing computational power, it is necessary to make changes and install patches for newly discovered security vulnerabilities. However, the process of installing updates requires a high level of access that is more than the default permissions on the IoT device. Most of these devices have maintenance mode intended for technicians in order to install updates and new software. Meaning that if the device users are not technically trained, it will probably be hard to carry out maintenance on their devices.

\section{Robust security}

When data is distributed across a large network while numerous data centers and devices are operating far from the main location, network visibility and control become a challenge. Each device represents another potentially vulnerable endpoint; therefore Internet of Things (IoT) lacks robust security[6]. Small data centers or server setups also have a security architecture that is not well designed, and these devices are not often updated as often as they should be.

\section{Loopholes in edge security}

Loopholes provide hackers easy access to the core of a network. Loophole challenges occur when IoT devices are rushed into the market before thorough testing is carried out. Also, loopholes are common to companies that rush to adopt technology without fully understanding the security risks involved[7].

\section{E. Communication}

Mostly, IoT devices are referred to as appliances since the data that they collect and transmit is considered innocuous, and it is mostly sent [2]. At the same time, it is not encrypted or authenticated. This is one of the biggest IoT security challenges since the data that seems innocuous could still be a value to attacker. Therefore encrypting communication increases the bar for hackers when attempting to learn the command and control syntax in order to take control of the device[10].

\section{F. Physical tampering}

Edge computing is a distributed technology, and this makes it vulnerable to physical risks. Also, servers and devices that power traditional networks are mostly housed in dedicated, often highly secure facilities, and these networks make up edge processing; thus, security can be a nightmare. Today, instead of data centers being housed in one location, micro-center devices are often deployed into various locations, which is IoT edges. This creates a risk since attackers can physically tamper with the edge devices to bring the network down or even harm the operators. Although hardening them against physical attack is costly, frequent servicing and upgrading edge data centers is necessary[9].

\section{G. Malicious hardware/ software injection}

This is also a common issue of edge computing on IoT devices, where attackers add authorized software or hardware into the communication network. This injects malicious input into the servers, exploiting service providers, creating a chance for hacking processes, including stealing data, reporting false, bypassing authentication, or even exposing database integrity. Hardware injections attacks can be classified into node replication, hardware trojan, camouflage, and corrupted or malicious EC nodes [4]. Node replication occurs when adversaries add a new malicious code to a network and assign an ID number similar to that of an authorized node. This allows attackers to misdirect data packets sent through the replica node. Hardware trojan is unauthorized access to integrated circuits, making attackers control and access data running through integrated circuits [11] [12].

\section{SOLUTION}

There are various practices that will ensure security at the edge of the network. The main solution is implementing end-to-end encryption where a long-term plan is deployed for an edge computing that will account for future use cases and compatibility of components and applications (Russell \& Duren, 2018). Securing devices with strong passwords is also a solution to the above security issues. IoT systems should also maintain authentication sessions of edge users through realtime identity monitoring. Corporate security services should analyze network logs in order to identify unknown devices within the organization's network parameter.

\section{CONCLUSION}

Edge computing has many promises, but it requires the users to put more effort and time into addressing and considering the security implications. Securing the edge of networks is also a complicated process. It requires network engineers and experts to use traditional security principles to enhance threat detection for the edge to automated patching for firmware and software. The above security issues affecting edge computing-based IoT applications will need to be resolved if edge computing is to be seen as an alternative to cloud computing. If these issues are resolved, then edge computing is expected to undergo explosive growth over the next decade, being bolstered by the Internet of Things technology also. It is expected that Industrial Internet Consortium is developing the Industrial Internet of Things device security, and this shows progress in certain edge computing areas. 


\section{REFERENCES}

[1] Cao, J., Zhang, Q., \& Shi, W. (2018). Edge computing: A primer. Springer.

[2] Abbas, N., Zhang, Y., Taherkordi, A., \& Skeie, T. (2018). Mobile edge computing: A survey. IEEE Internet of Things Journal, 5(1), 450-465. doi:10.1109/jiot.2017.2750180

[3] Al-Turjman, F. (2018). Edge computing: From hype to reality. Springer.

[4] Asad, M. M. (2016). Investigating security challenges and issues for Internet of things (IoT) industrial applications.

[5] Jeyanthi, N., Banthia, S., \& Sharma, A. (2017). Security in IoT devices. Security Breaches and Threat Prevention in the Internet of Things, 96116. doi:10.4018/978-1-5225-2296-6.ch005

[6] Tadapaneni, N. R. (2019). Role of Fog Computing in the Internet of Things. International Journal of Scientific Research and Engineering Trends.
[7] Tadapaneni, N. R. (2018). Cloud Computing: Opportunities And Challenges. International Journal of Technical Research and Applications.

[8] Ambrose, T. V., Chidhambaran, R., \& Mohan, M. C. Technological changes: Recent trends and challenges in banking sector.

[9] Russell, B., \& Duren, D. V. (2018). Practical Internet of things security: Design a security framework for an internet connected ecosystem (2nd ed.). Packt Publishing.

[10] Qazi, R., \& Khan, I. A. (2020). Data Security in Cloud Computing Using Elliptic Curve Cryptography.

[11] Tadapaneni, N. R. (2020). Cloud Computing - An Emerging Technology. International Journal of Innovative Science and Research Technology. 5.

[12] Alhenaki, L. M., Bashaer, A., Alamri, A., \& Alarifi, N. (2019). A Survey on the Cloud Computing Security. In International Conference on Computer Applications \& Information Security (May, 2019). 